\title{
Kolokosides A-D: Triterpenoid Glycosides from a Hawaiian Isolate of Xylaria sp. ${ }^{\perp}$
}

Stephen T. Deyrup, ${ }^{\dagger}$ James B. Gloer, ${ }^{\dagger *}$ Kerry O’Donnell, ${ }^{\ddagger}$ and Donald T. Wicklow

Department of Chemistry, University of Iowa, Iowa City, Iowa 52242 and Mycotoxin Research Unit, Agricultural Research Service, National Center for Agricultural Utilization Research, USDA, Peoria, Illinois 61604

\section{List of Supporting Information}

Figure S1. ${ }^{1} \mathrm{H}$ NMR spectrum of kolokoside $\mathrm{A}\left(1 ; \mathrm{CD}_{3} \mathrm{OD}, 600 \mathrm{MHz}\right)$

Figure S2. ${ }^{13} \mathrm{C}$ NMR spectrum of kolokoside $\mathrm{A}\left(\mathbf{1} ; \mathrm{CD}_{3} \mathrm{OD}, 100 \mathrm{MHz}\right)$

Figure S3. ${ }^{1} \mathrm{H}$ NMR spectrum of kolokoside $\mathrm{B}\left(2 ; \mathrm{CD}_{3} \mathrm{OD}, 600 \mathrm{MHz}\right)$

Figure S4. ${ }^{13} \mathrm{C}$ NMR spectrum of kolokoside $\mathrm{B}\left(2 ; \mathrm{CD}_{3} \mathrm{OD}, 100 \mathrm{MHz}\right)$

Figure S5. ${ }^{1} \mathrm{H}$ NMR spectrum of kolokoside $\mathrm{C}\left(3 ; \mathrm{CD}_{3} \mathrm{OD}, 600 \mathrm{MHz}\right)$

Figure S6. ${ }^{13} \mathrm{C}$ NMR spectrum of kolokoside $\mathrm{C}\left(\mathbf{3} ; \mathrm{CD}_{3} \mathrm{OD}, 100 \mathrm{MHz}\right)$

Figure S7. ${ }^{1} \mathrm{H}$ NMR spectrum of kolokoside D (4; $\left.\mathrm{CD}_{3} \mathrm{OD}, 600 \mathrm{MHz}\right)$

Figure S8. ${ }^{13} \mathrm{C}$ NMR spectrum of kolokoside D $\left(4 ; \mathrm{CD}_{3} \mathrm{OD}, 100 \mathrm{MHz}\right)$

Figure S9. ${ }^{1} \mathrm{H}$ NMR spectrum of 5,6-epoxycytochalasin N (acetone- $d_{6}, 400 \mathrm{MHz}$ )

${ }^{\perp}$ Dedicated to the late Dr. Kenneth L. Rinehart of the University of Illinois at Urbana-Champaign in honor of his pioneering work on bioactive natural products.

*To whom correspondence should be addressed. Tel: 319-335-1361. Fax: 319-335-1270. Email: james-gloer@uiowa.edu.

$\dagger$ University of Iowa

$¥$ USDA 
Figure S1. ${ }^{1} \mathrm{H}$ NMR spectrum of kolokoside $\mathrm{A}\left(1 ; \mathrm{CD}_{3} \mathrm{OD}, 600 \mathrm{MHz}\right)$

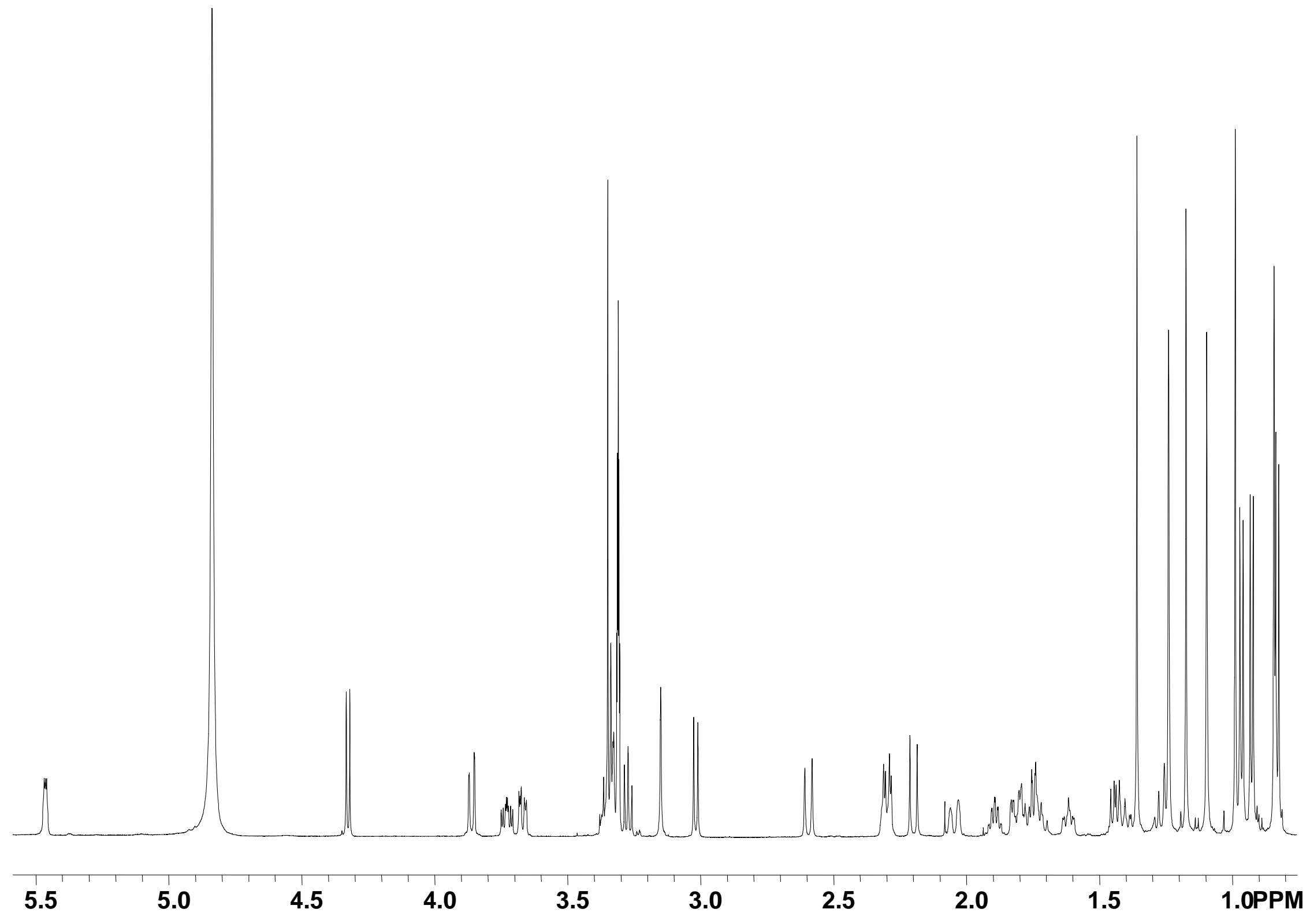


Figure S2. ${ }^{13} \mathrm{C}$ NMR spectrum of kolokoside $\mathrm{A}\left(1 ; \mathrm{CD}_{3} \mathrm{OD}, 100 \mathrm{MHz}\right)$

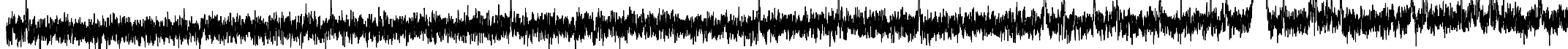


Figure S3. ${ }^{1} \mathrm{H}$ NMR spectrum of kolokoside $\mathrm{B}\left(2 ; \mathrm{CD}_{3} \mathrm{OD}, 600 \mathrm{MHz}\right)$

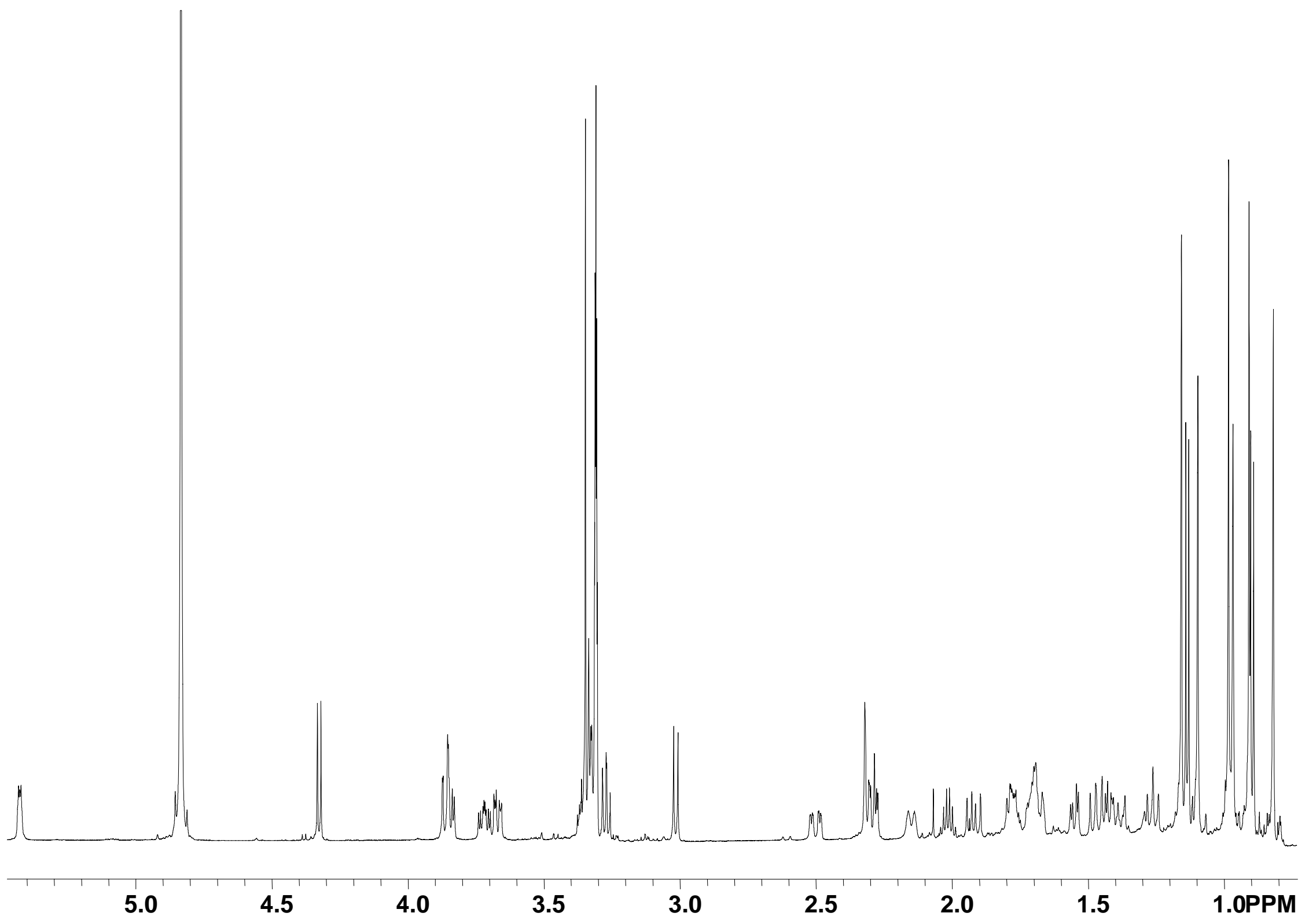


Figure S4. ${ }^{13} \mathrm{C}$ NMR spectrum of kolokoside $\mathrm{B}\left(2 ; \mathrm{CD}_{3} \mathrm{OD}, 100 \mathrm{MHz}\right)$

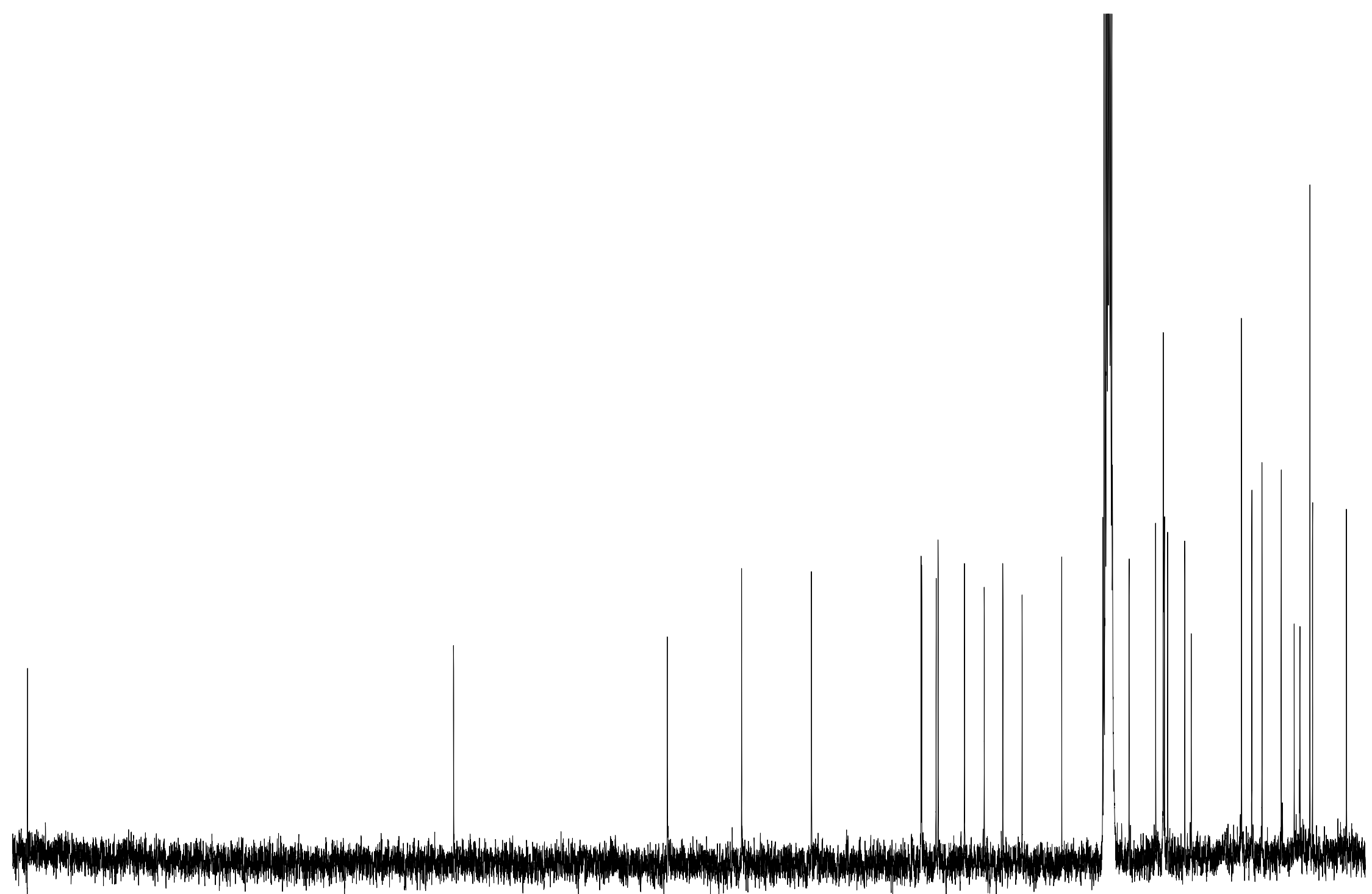


Figure S5. ${ }^{1} \mathrm{H}$ NMR spectrum of kolokoside $\mathrm{C}\left(3 ; \mathrm{CD}_{3} \mathrm{OD}, 600 \mathrm{MHz}\right)$

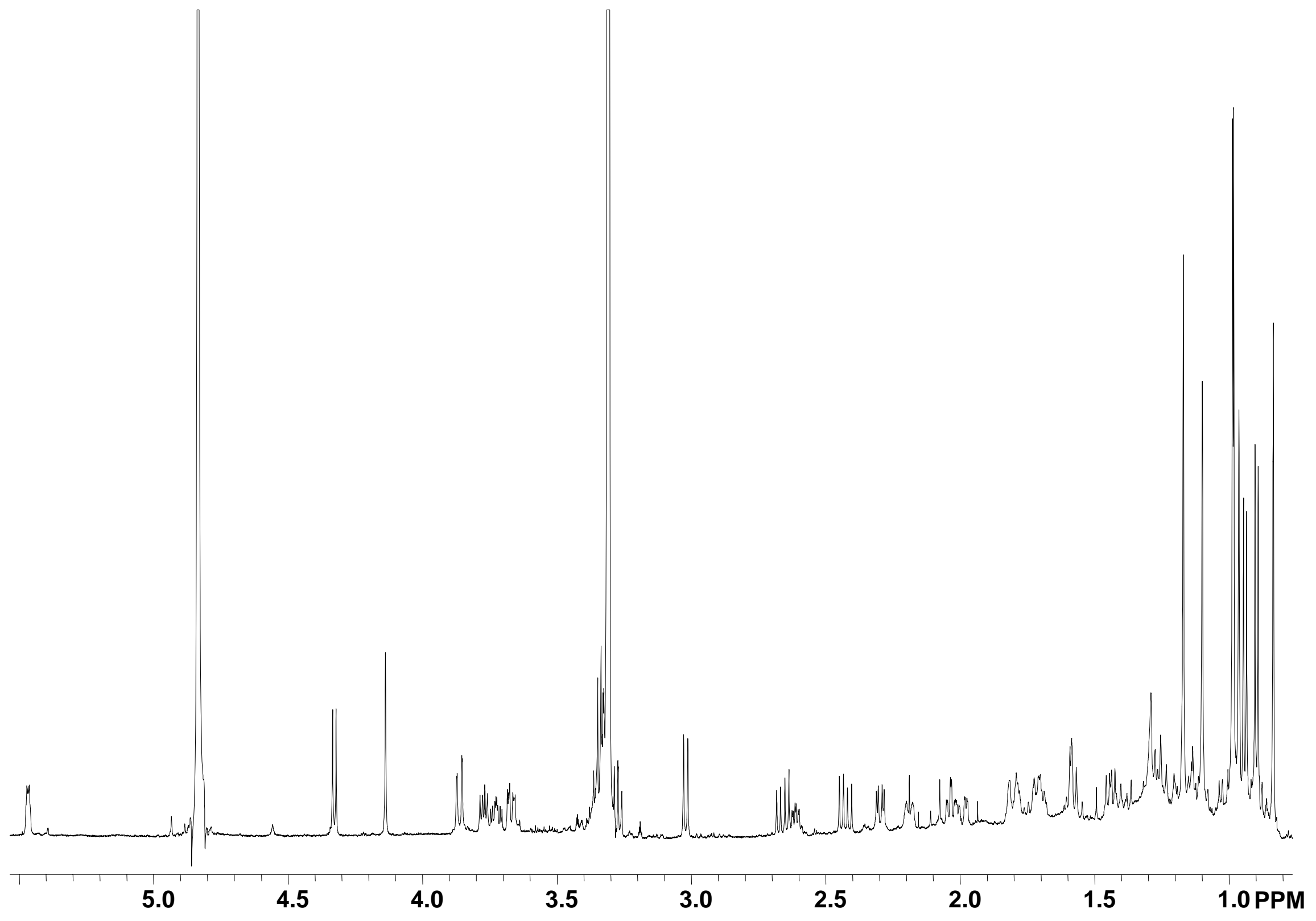


Figure S6. ${ }^{13} \mathrm{C}$ NMR spectrum of kolokoside $\mathrm{C}\left(3 ; \mathrm{CD}_{3} \mathrm{OD}, 100 \mathrm{MHz}\right)$

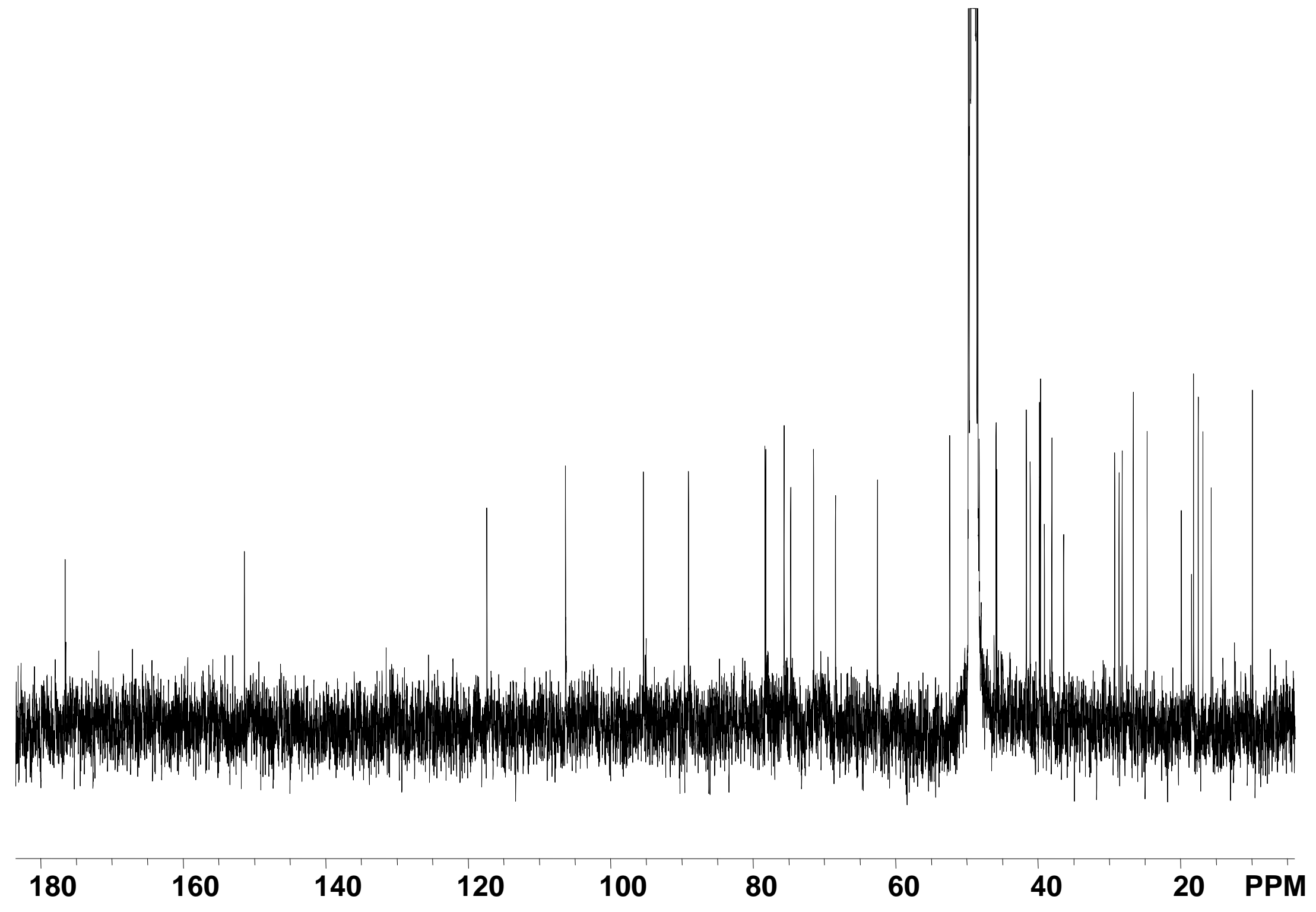


Figure S7. ${ }^{1} \mathrm{H}$ NMR spectrum of kolokoside $\mathrm{D}\left(4 ; \mathrm{CD}_{3} \mathrm{OD}, 600 \mathrm{MHz}\right)$

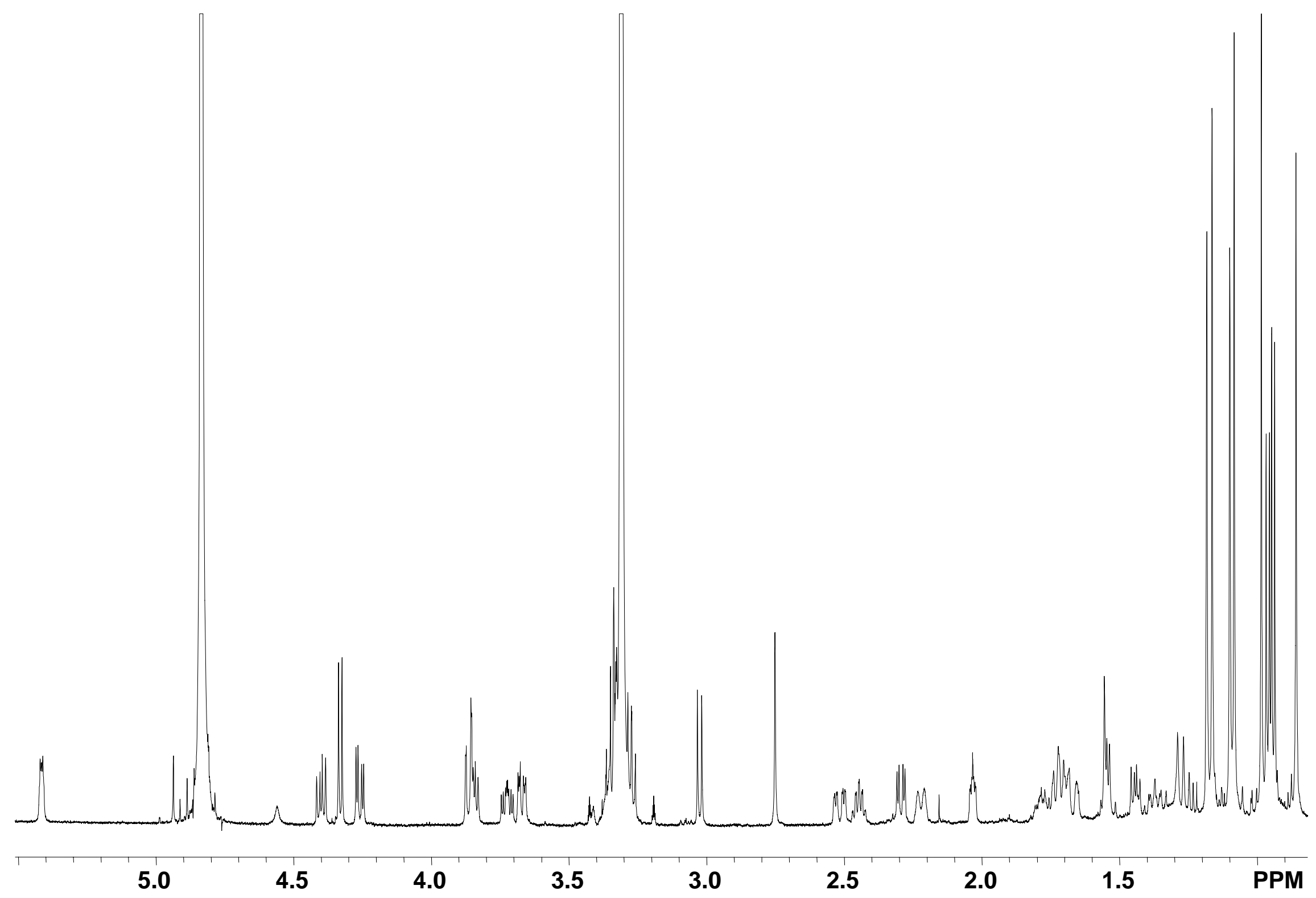


Figure S8. ${ }^{13} \mathrm{C}$ NMR spectrum of kolokoside $\mathrm{D}\left(4 ; \mathrm{CD}_{3} \mathrm{OD}, 100 \mathrm{MHz}\right)$

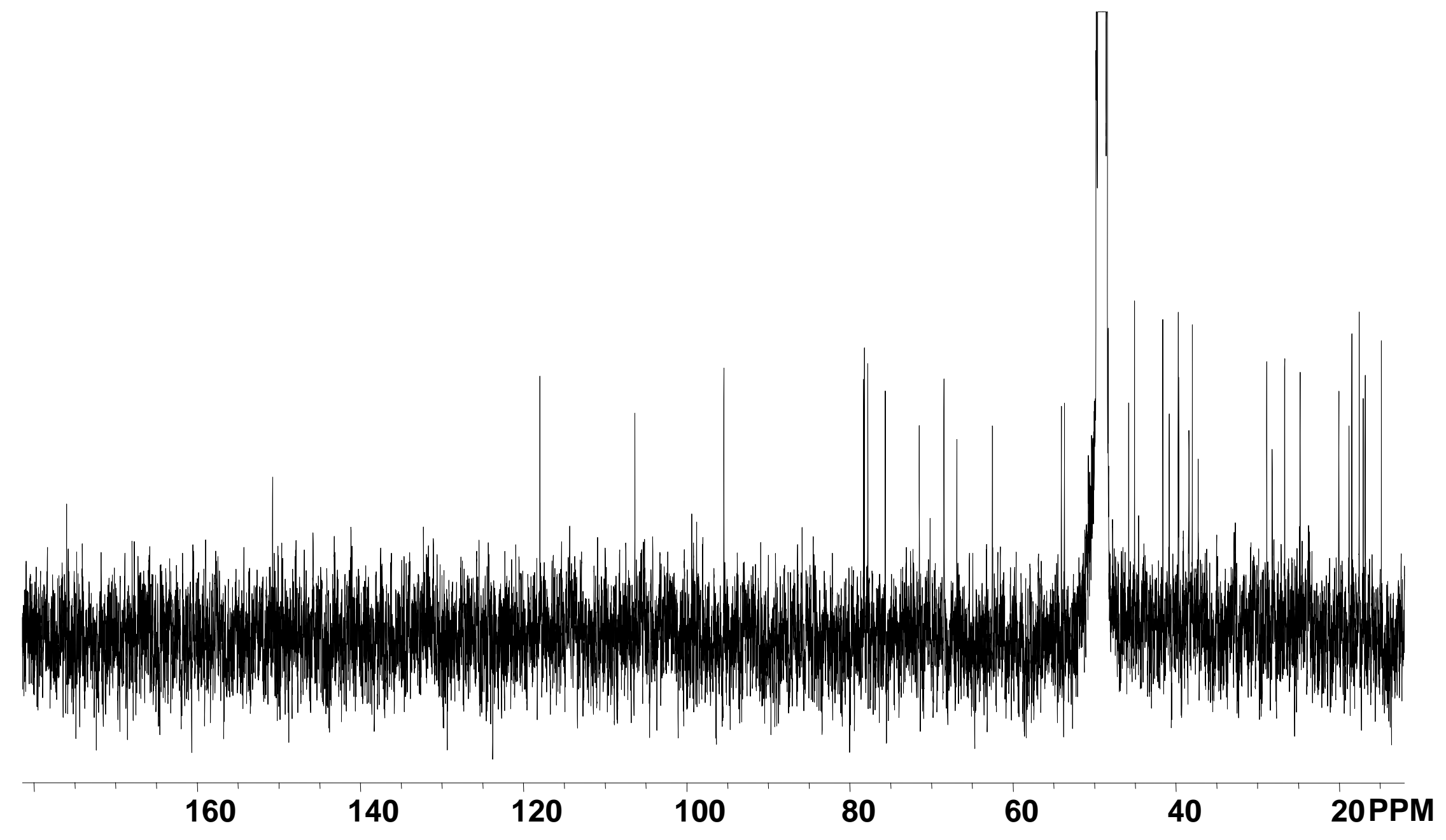


Figure S9. ${ }^{1} \mathrm{H}$ NMR spectrum of 5,6-epoxycytochalasin N (acetone- $d_{6}, 400 \mathrm{MHz}$ )

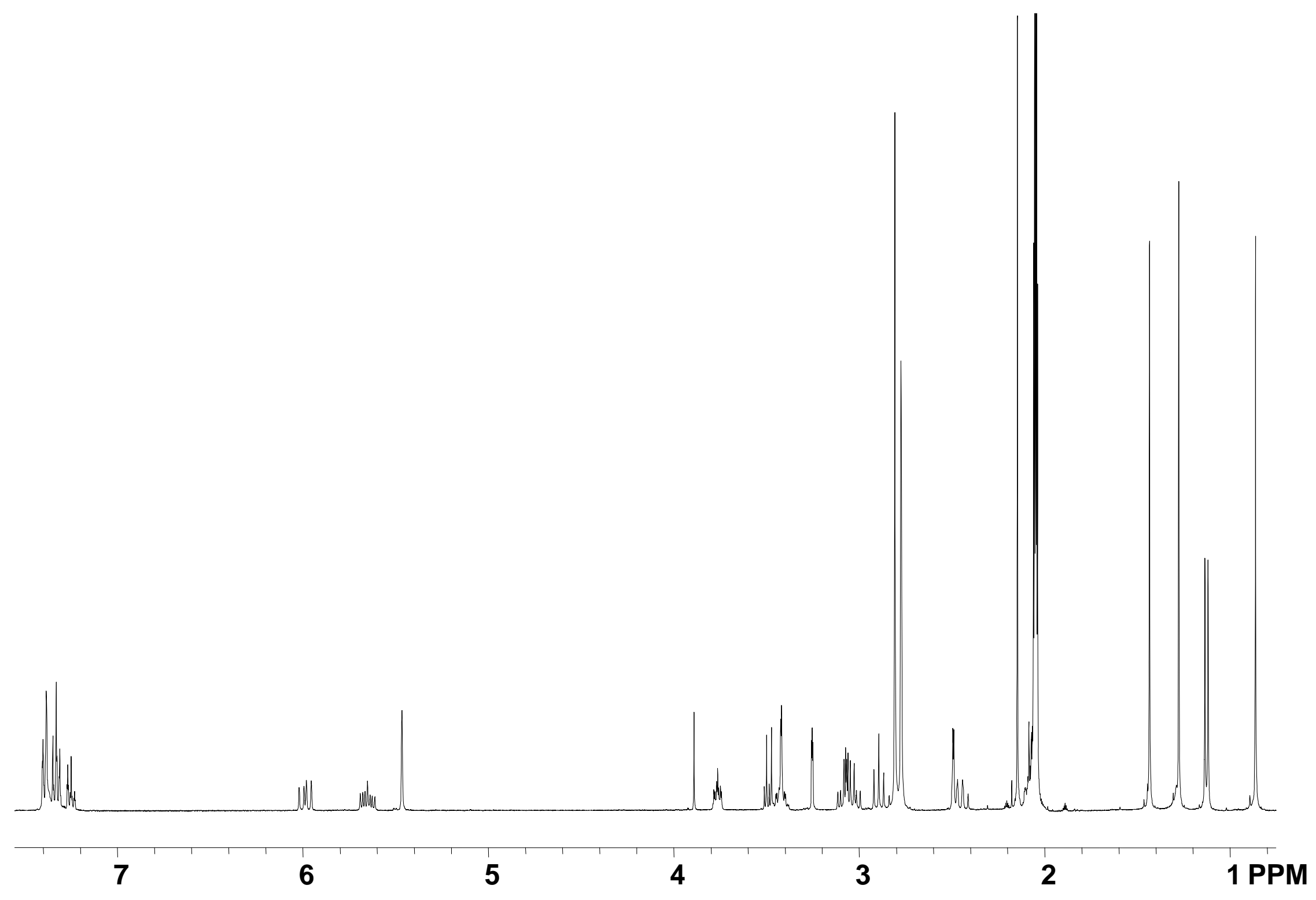

